\title{
Computational Studies of the Corrosion Inhibition Potentials of Quercetin and Coumarin
}

\author{
VM Udowo* \\ Department of Science, Topfaith International Secondary School, Nigeria
}

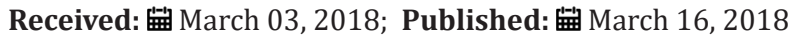

*Corresponding author: VM Udowo, Department of Science, Topfaith International Secondary School, Nigeria, Tel: 2348039454661;

Email: Victorudowo@gmail.com

\begin{abstract}
The molecules of Coumarin and Quercetin were subjected to computational calculations using the Density Functional Theory (DFT) in order investigate their inhibition potentials. The Coumarin molecule demonstrated a greater corrosion inhibition potentials compared to the Quercetin molecule. The corrosion potentials were predicted using quantum chemical parameters like Energy of the highest occupied molecular orbital (EHOMO) and Energy of the lowest unoccupied molecular orbital ( $\mathrm{E}_{\text {Luмо }}$ ), ionization potential (I), Fukui ( $\mathrm{f}^{+}$and $\mathrm{f}^{-}$) indices and Global hardness.
\end{abstract}

\section{Introduction}

The Computational study was carried out to calculate the electronic parameters for the Coumarin and Quercetin molecules. The following quantum chemical indices were considered to research the relationship of molecular structure and inhibition effect: the energy of the highest occupied molecular orbital (EHOMO), the energy of the lowest unoccupied molecular orbital (ELUMO), energy band gap $\triangle \mathrm{E}=\mathrm{EHOMO}-$ ELUMO, the electro negativity $(\chi)$, global hardness $(\eta)$, global electrophilicity $(\omega)$, global softness $(\sigma)$, and the change in the number of electrons transferred $(\Delta \mathrm{N})$, electron affinity, ionization potential, molecular weight, total

dipole moment, Van der Waal's surface area. According to frontier molecular orbital theory, the chemical reactivity is a function of interaction between HOMO and LUMO levels of reacting species [1]. Theoretical calculations were conducted in order to provide molecular-level understanding of the corrosion inhibition behavior of Coumarin and Quercetin inhibitor. (Figures 1a \& 1b) show the optimized structures, and (Figures 2a \& 2b) show the HOMO and LUMO orbitals diagrams of Coumarin and Quercetin molecules respectively. The above listed parameters were considered to be of great importance in studying the chemical interaction between the inhibitors and the metal surface.

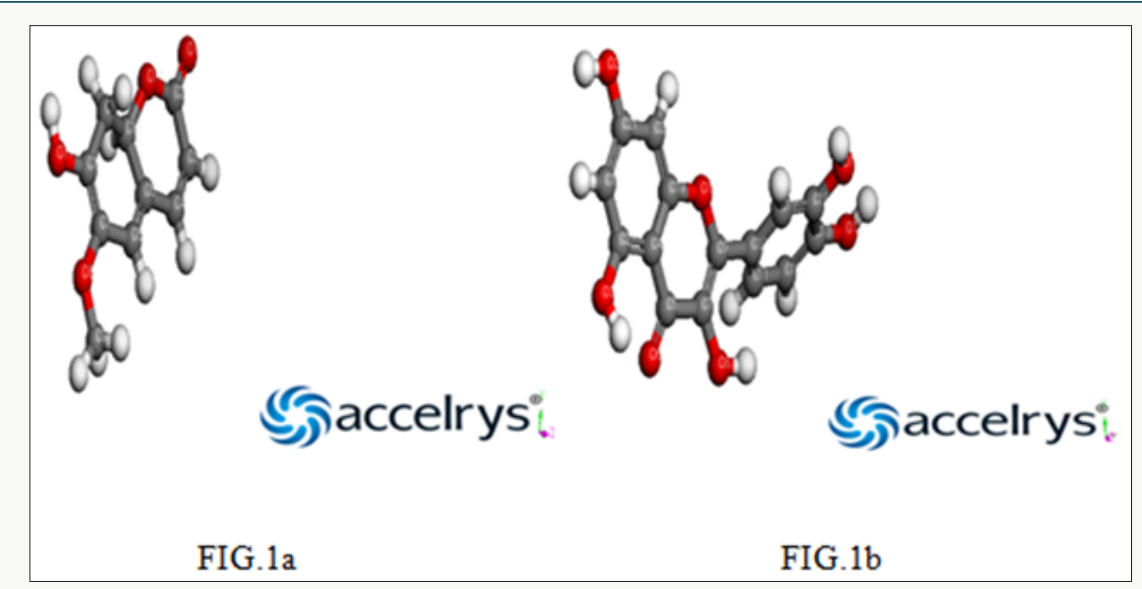

Figure 1a \& 1b: Show the geometric optimized structure of coumarin and quercetin. 


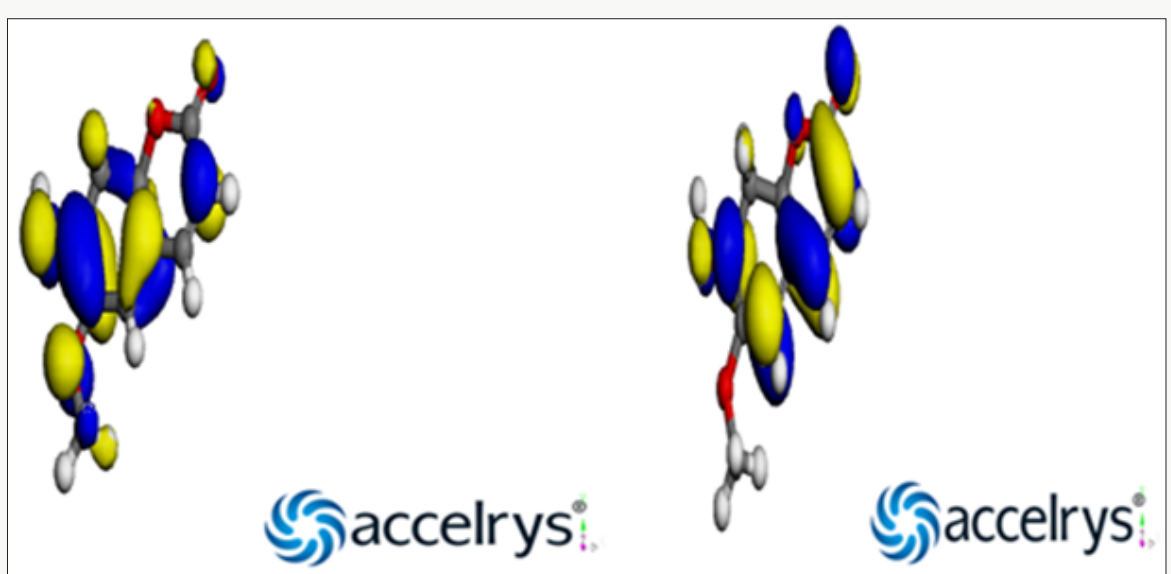

Figure 1a \& 1b: Show the geometric optimized structure of coumarin and quercetin.

\section{Computational Details}

\section{Global reactivity}

Quantum chemical calculation is one of the widely used tools in corrosion studies [2]. In this work, different quantum chemical parameters were calculated and the results obtained from the DFT calculations were presented in Table 1 . The most significant parameters in the semi empirical result are the frontier molecular energies, which are the energy of the highest occupied molecular orbital $\left(\mathrm{E}_{\text {номо }}\right)$, the energy of the lowest unoccupied molecular orbital $\left(\mathrm{E}_{\text {Luмо }}\right)$ and the band energy gap $(\Delta \mathrm{E})$. The $\mathrm{E}_{\text {номо }}$ correlates with the electron donating capacity of the specie. A higher value of $\mathrm{E}_{\text {ном }} \mathrm{O}$ is thus associated with better inhibitor [3]. On the other hand $\mathrm{E}_{\mathrm{Lumo}}$ is connected with the affinity of the specie to accept electron [4]. Thus, the lower value of $\mathrm{E}_{\text {Lumo }}$ implies better efficiency. The energy gap of a molecule $\Delta \mathrm{E}$ is related with the hardness and softness of the specie [5]. Larger energy gap implies that the molecule will need much energy to move from HOMO to the LUMO. Therefore, larger energy gap shows that the molecule is hard, while lower energy gap points toward soft molecule [6]. From the results presented in (Table 1), it is shown that quercetin is likely to be a better inhibitor than coumarin. This is because the $\mathrm{E}_{\text {номо }}$ value of coumarin is relatively higher than that of quercetin. Similarly the $\mathrm{E}_{\text {Lumo }}$ value of coumarin is higher than quercetin and $\Delta \mathrm{E}$ value of coumarin is higher than that of quercetin.

Table 1: Quantum chemical parameters for the studied chemical constituents of Coumarin and Quercetin.

\begin{tabular}{|c|c|c|}
\hline Parameters & Coumarin & Quercetin \\
\hline Electronegativity $\chi(\mathrm{eV})$ & 4.0185 & 1.04 \\
\hline Global Hardness $\eta(\mathrm{eV})$ & 1.004 & 0.705 \\
\hline Global softness $\sigma(\mathrm{eV}-1)$ & 0.995 & -5.457 \\
\hline EHOMO $(\mathrm{eV})$ & -5.023 & -2.624 \\
\hline ELUMO $(\mathrm{eV})$ & -3.014 & -8.081 \\
\hline Energy Gap $\Delta \mathrm{E}(\mathrm{eV})$ & -8.037 & 5.762 \\
\hline Global electrophilicity $\omega(\mathrm{eV}-1)$ & 8.037 & 2.624 \\
\hline Electron affinity & 3.014 & -1.044 \\
\hline Change in Electron's number Transferred $\Delta \mathrm{N}$ & -1.484 & 302.24 \\
\hline Molecular Weight & 194.18 & 291.45 \\
\hline Van der Waal's Forces & 211.63 & 4.226 \\
\hline Total dipole moment & 3.172 & 5.457 \\
\hline Ionization potaential & 5.023 & \\
\hline
\end{tabular}

Electronegativity $(\chi)$ is the measure of the power of an electron or group of atoms to attract electrons towards it [7]. The calculated electronegativity values presented in (Table 1), show that quercetin has higher $\chi$ value than coumarin. This implies that quercetin has a higher power to attract electrons to itself and hence a better inhibitor than coumarin. (Figure 3a \& 3b) Global softness ( $\sigma$ ) and global hardness ( $\eta$ ) values are equally reported in (Table 1 ). Global softness describes the capacity of an atom or group of atoms to receive electrons [8]. Global hardness ( $\eta$ ) measures the resistance of an atom to a charge transfer [9]. The results obtained for the $\sigma$ value of Coumarin is higher than the value obtained for quercetin. While the $\eta$ value for quercetin is higher than that obtained for coumarin. This implies that coumarin is a softer molecule than quercetin and it is expected to be a better inhibitor. A high value of electrophilicity 
describes a good electrophile while a small value of electrophilicity describes a good nucleophile [1]. The calculated electrophilicity values reported in (Table 1), shows that coumarin has a higher value when compared with the value obtained for Quercetin. Hence coumarin is a better electrophile as well as a better inhibitor when compared with Quercetin.

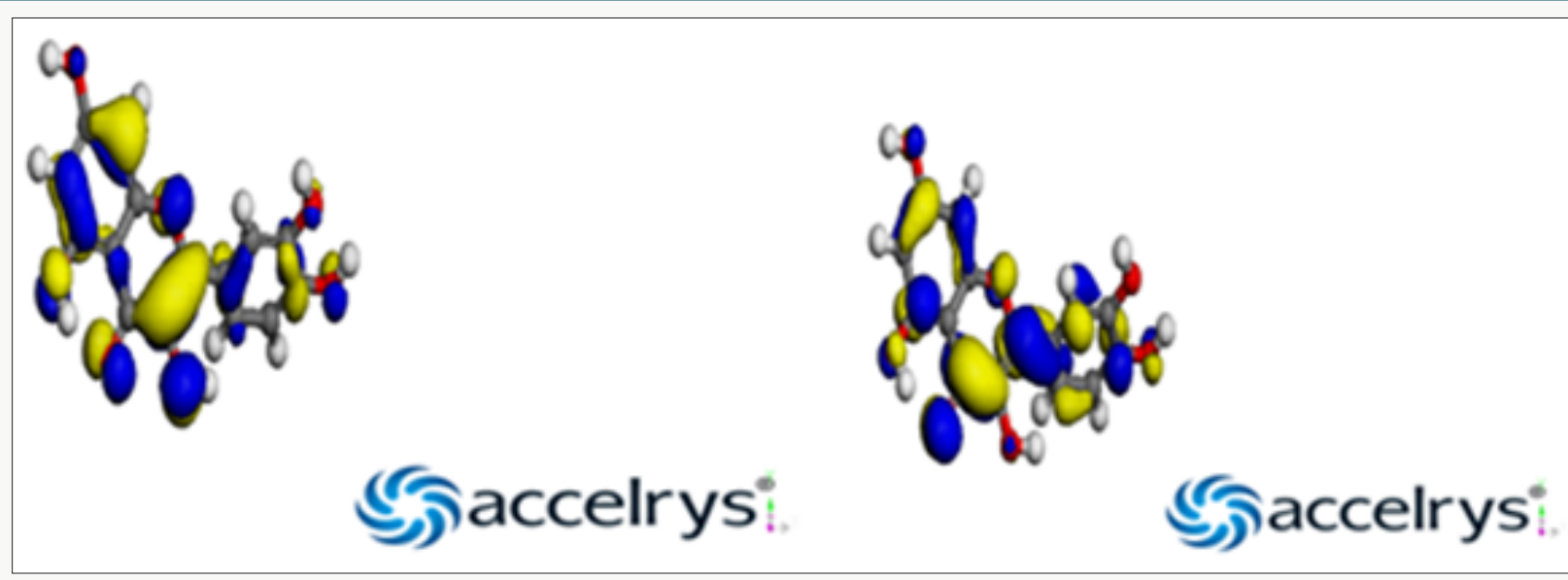

Figure 3a \& 3b: Homo and Lumo Diagrams for Quercetin.

The difference in the number of electrons transferred between the retarder and the adsorbent (Fe) is represented using $\Delta \mathrm{N}$ [10]. The $\Delta \mathrm{N}$ values goes to tell the likelihood of a molecule to give out electrons [11]. A high $\Delta \mathrm{N}$ value shows a greater tendency for a molecule to donate electrons to the poor electron species and interact with the metal surface (a greater chance of adsorption) [12]. From the calculated results in (Table 1), quercetin has a higher $\Delta \mathrm{N}$ value compared to coumarin. This implies that quercetin is a better inhibitor than coumarin. Other calculated parameters include: dipole moment which provides information on the polarity of the molecule and it is also a good reactivity indicator. Molecular weight indicates the possible metal surface coverage by the inhibitor. The larger the molecular weight of an inhibitor, the greater the ionization energy because large molecule volume implies increased surface coverage [13]. Molecular weight indicates possible metal coverage. Therefore large the molecular weight values indicate better inhibition efficiencies. The molecular weight value of quercetin is higher than that of coumarin. Hence quercetin is a better inhibitor than coumarin.

\section{References}

1. Ebenso EE, Kabanda MM, Muralana LC, Ozcan M, Karadag F, et al. (2012) Quantum Chemical studies on the corrosion inhibition of mild steel by some Triazoles and Benzimidazole Derivatives in Acidic Medium. International Journal of Electrochemical Science 7: 5035-5056.

2. Eddy NO, Ita BI (2011b) Theoretical and experimental studies on the inhibition potentials of aromatic oxaldehydes for the corrosion of mild steel in 0.1 M HCl. Journal of Molecular Modelling 17(4): 633-647.

3. Eddy NO, Ita BI (2011a) Experimental and theoretical studies on the inhibition potentials of some derivatives of cyclopenta-1,3-diene for the corrosion of mild steel in $\mathrm{HCl}$ solutions. International Journal of Quantum Chemistry 111(14): 3456-3473.
4. Jensen F (2007) Introduction to computational chemistry, John Wiley \& sons, Chichester.

5. Udowo VM, Uwah IE, Daniel EF, Abeng FE, Ivara S (2017) Computational and experimental study of the inhibition effects of purple sweet potato leaves extract on mild steel corrosion in $1 \mathrm{M} \mathrm{H} 2 \mathrm{SO}$. Journal of physical chemistry and biophysics 7:253.

6. Ebenso EE, Obot IB (2010) Inhibitive properties, thermodynamic characterization \& quantum chemical studies of secnidazole on mild steel corrosion in acidic medium. International Journal of Electrochemical Science 5: 2012-2035.

7. Young DC (2004) Computational Chemistry: A practical guide for applying techniques to real world problems. Wiley interscience, New York, USA.

8. Eddy NO (2010) Theoretical study on some amino acids \& their potential activity as corrosion inhibitors for mild steel in $\mathrm{HCl}$. Molecular Simulation 35(5): 354-363.

9. Eddy NO (2011) Experimental \& theoretical studies on some amino acids and their potential activity as inhibitors for the corrosion of mild steel. Journal of Advanced Research 2: 35-47.

10. Afia L, Larouj R, Salghi S, Jodeh S, Zougagh M, et al. (2016) Experimental and theoretical evaluation of quercetin as a novel and eco-friendly inhibitor for C38 Steel in HCl medium. Der Pharma Chemica 8(3): 166179.

11. Obot IB, Obi Egbeidi (2010) Adsorption properties \& inhibition of mild steel corrosion in sulphuric acid solution by ketoconazole: experimental \& theoretical investigation. Corrosion Science 52(1): 198-204.

12. Gao G, Liang C (2007) Electrochemical \& DFT studies of beta-amino alcohols as corrosion inhibitors for brass. Electrochemical Acta 52(13): 4554-4559.

13. Awe FE, Idris SO, Abdulwahab M, Oguzie EE (2015) Theoretical and experimental inhibitive properties of mild steel in $\mathrm{HCl}$ by ethanolic extract of Boscia senegalensis. Cogent Chemistry 7(9): 12676. 
(C) Comons Attribution 4.0 License

To Submit Your Article Click Here: Submit Article

DOI: 10.32474/AOICS.2018.02.000133

\section{AOICS}

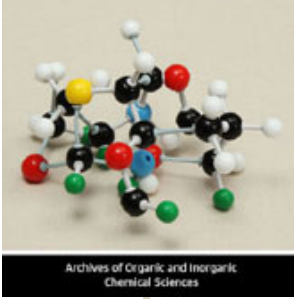

Archives of Organic and Inorganic Chemical Sciences

\section{Assets of Publishing with us}

- Global archiving of articles

- Immediate, unrestricted online access

- Rigorous Peer Review Process

- Authors Retain Copyrights

- Unique DOI for all articles 